\title{
FRACTALITY OF MEASUREMENTS OF QUANTITIES AND REAL PROCESSES
}

\author{
Nona Otkhozoria, PhD, Georgian Technical University, Georgia, Tbilisi \\ Vano Otkhozoria, PhD, Georgian Technical University, Georgia, Tbilisi \\ Medea Narchemashvili, PhD, Georgian Technical University, Georgia, Tbilisi
}

DOI: https://doi.org/10.31435/rsglobal_conf/30062021/7620

\begin{abstract}
In the present work, the measurement of quantities and time processes from the point of view of their fractal properties is considered, which implies the calculation of the dimension proposed by Mandelbrot in two ways: the first is the practical definition of the fractal dimension based on the calculation of the correlation dimension, the second is the empirical determination of the fractal dimension by the method of the normalized Hurst range. Experimental results were obtained using Labview and Mathcad technologies. experimental studies support the persistence in measuring dynamic processes and the ability to predict the behavior of an object in the future. It was revealed that the second method for determining the fractal dimension is obvious and easy to implement. However, for this we have to deal with data describing the dynamics of the process, i.e. with a time series. In the article, using specific examples, the use of fractals in metrology is shown, in particular, in relation to the uncertainty of the measurement result.
\end{abstract}

Introduction. When it comes to fractals, it should first of all be emphasized that the idea of studying fractals arose due to the impossibility of measuring the length of the coastline of Norway. Measurements were carried out by various tools and methods offered by many scientists [1]. And only the introduction of fractals and fractal dimension made it possible to solve many problems and, in particular, to study the behavior of real-natural processes in terms of the possibility of predicting their behavior in the future [1-3]

Let's start by defining the fractal itself.

One of the definitions of fractal: fractal is a set, parts of which are similar to the whole. The principle of self-similarity is a characteristic determinant of the essence of fractals. The first most famous scientist to study fractals was B. Mandelbrot. In the mid-60s of the twentieth century, he developed fractal geometry, which he called the geometry of nature. He is the "father" of fractals, because he was the first to use them in the analysis of fuzzy, irregular forms. The idea embedded in fractals is in nontarget dimensions. We usually use integer dimensions - dimensions. However, non-integral dimensions may exist, for example, 2.58, i.e., between two-dimensional and three-dimensional measurements. Mandelbrot called such measurements - dimensions fractal (fractional, divided into parts) [1,2].

The logic of the existence of non-target dimensions is very simple. So, in nature there is no perfect ball or cube without scratches and other irregularities. And to describe such objects, there must be other dimensions. To measure such irregular, fractal figures, the concept of fractal measurement was introduced - fractal dimension [1-3].

For example, roll a sheet of paper into a lump. From the standpoint of classical Euclidean geometry, this object will be a three-dimensional ball in a three-dimensional dimension. However, in reality, this is still just a two-dimensional sheet of paper, crumpled in the likeness of a ball. Thus, it can be assumed that the new object will have a dimension of more than two, but less than three, i.e. it will be in the fractal dimension, i.e. it will have a dimension of about $2.5 \mathrm{pm}$. The physical meaning of this dimension means that in classical space there remains, due to the gaps and holes naturally present in the crumpled sheet of paper, part of the space. In his work [2], he showed that fractal dimension is the inverse of $\mathrm{H}$, where $\mathrm{H}$ is Hurst's index. So, if $\mathrm{H}=0.5$ (Brownian motion) $\mathrm{D}=2,(1 / 0.5)$, and at $\mathrm{H}=0.8$, the fractal dimension is $1.25(1 / 0.8)$.

But there is another fractal dimension D, [1] (according to Mandelbrot), which is defined as $\mathrm{D}=2-\mathrm{H}$, and this is an estimate of the degree of fracture of the time series, which will be discussed when assessing the persistence and antipersistency of actually observed data describing a dynamic process (Hearst's empirical law is the normalized scope of R/S) [1]. 
The purpose of this work is to consider the measurement of quantities and time processes from the point of view of their fractal properties, which involves calculating the dimension proposed by Mandelbrot in two ways: the first-practical definition of fractal dimension based on the calculation of correlation dimension, and then the Hurst index. Second application of Hearst normalized span (R/S) empirical law for direct measurement of $\mathrm{H}$ (Hearst index) followed by calculation of fractal dimension. The numerical value of $\mathrm{H}$ depends on the presence of a deterministic pattern in the object of measurement, and therefore the possibility of predicting its behavior in the future. The task was also to show, with specific examples, the use of fractals in metrology, in particular with regard to the uncertainty of the measurement result.

\section{Main part}

\subsection{Mathematical interpretation}

How to imagine the dimension of fractals mathematically? Let to fill an arbitrary geometric structure with a Euclidean dimension $D_{E}$ require $\mathrm{N}$ (a) circles for flat figures with a diameter? and spheres for figures in space (in a three-dimensional dimension). When dimensioned $(\varepsilon \rightarrow 0)$ the sum of the circles tends to the volume occupied by the geometric object. In this case, the fractal dimension $\left(D_{F}\right)$ is defined as

$$
D_{F}=\lim _{\varepsilon \rightarrow 0} \frac{\ln N(\varepsilon)}{\ln (1 / \varepsilon)}
$$

Follows from expression (1) that for a set of the points forming the smooth line, Euclidean $D_{E}=D_{F}=1$. At the same time the structure of a geometrical object will be fractal if $D_{E} \neq D_{F}$. Clearly, $D_{E}$ always takes only integer values. Therefore, fractal dimension is non-numeric and characterizes objects with a complex structure.

In practical fractal dimensionality calculations, the correlation dimensionity $D_{C}$ is used, which is determined by the expression:

$$
D_{C}=\lim _{\varepsilon \rightarrow 0} \frac{\ln C(\varepsilon)}{\ln (\varepsilon)}
$$

where $(\varepsilon)$ is a correlation function calculated as:

$$
\mathrm{C}(\varepsilon)=\lim _{N \rightarrow 0} \frac{n}{N^{2}}
$$

It follows from the expression (3) that the value of $(\varepsilon)$ is calculated as the ratio of the number of points $n$, the pairwise distances between which are less than sound, to the square of the total number of points N. Accordingly, in order to determine the correlation dimension $D_{C}$ first of all, it is necessary to calculate the number of points, the pairwise Euclidean distances (d) between which are less than the given distance?

$$
d=\sqrt{\left(x_{2}-x_{1}\right)^{2}+\left(y_{2}-y_{1}\right)^{2}}
$$

where $x_{1}, y_{1}$ - are the coordinates of point 1 , and $x_{2}, y_{2}$ - are the coordinates of point $\mathrm{d} \leq \varepsilon$.

Note that all the calculations of the fractal dimension of research objects presented below are implemented by Mathcad and LabVIEW technologies, and are a powerful mathematical tool for fractal research. In the present article it will be a question of application of fractals in the sphere of measurements (metrology) and, in particular, the modern concept of measurement uncertainty (types) and also at measurement and the analysis of temporary dynamic processes. (Heisenberg and Brillouin uncertainty principle) [5].

The relationship of the fractal to the uncertainty of measurements is easy to see. No matter how accurately we measure, we cannot approach the true value, and the reason is: firstly, due to the error of the standard itself, secondly, due to the influence of the measurement conditions, thirdly, due to the change in the measurement object itself (the principle of indeterminacy of Heisenberg and Brillouin).

It is impossible to talk about the size measured by the caliper, that it is equal to $24.5 \pm 0.05 \mathrm{~mm}$. Metrologically, it is more correct to represent this size in the form of an area from 24.50 to $24.60 \mathrm{~mm}$. If the same size is measured with a micrometer, then the boundaries of the result will be from $24.554 \mu \mathrm{m}$ to $24.558 \mu \mathrm{m}$. The uncertainty region decreases $(0.004)$.

Let's say that we measure the same parameter by the verified measuring means: first by the working measuring means, then by the working standard, then by the standard of the 2 nd digit, then by the standard of the 1st digit and, finally, by the main standard.

In this case, this process is similar to the fractal formation process. With each step, we get a decreasing measurement uncertainty, that is, the blur of the measurement result decreases. In work [4], the question of uncertainty is considered when assessing the quality of measurements. It should be noted that the fractal approach can be effective in applying uncertainty type B [5]. 
In the LabVIEW software, for example, [6] provides an example of measuring weights.

Our purpose is to define the fractal dimension offered by Mandelbrot, two ways of her definition $-D_{F}$.

In the first case, the dimension is calculated directly, and Hurst $\mathrm{H}$ is described by $\mathrm{H}=1 / D_{F}$, and in the second case $D_{F}=2-\mathrm{H}$ when $\mathrm{H}$ is determined experimentally. In both cases, the numerical value of $\mathrm{H}$ is necessary to judge the persistence and antipersistence of the process [1-3].

\subsection{Determination of fractal dimension by method 1}

When directly calculating the fractal dimension (method 1), the work consisted at the first stage in modeling a random variable distributed according to a uniform law, and at the second stage in processing real data obtained to determine the amount of gold (quartzite) in the ore. These works were carried out by Company in an open career way. The first figure shows the ore quarry, and the second figure 2, for example, a table containing the presence of quartzite (gold) in ore and without gold (K) in ore. According to the data obtained in a certain time, fractal processing was carried out (the first method of determining fractal dimension).

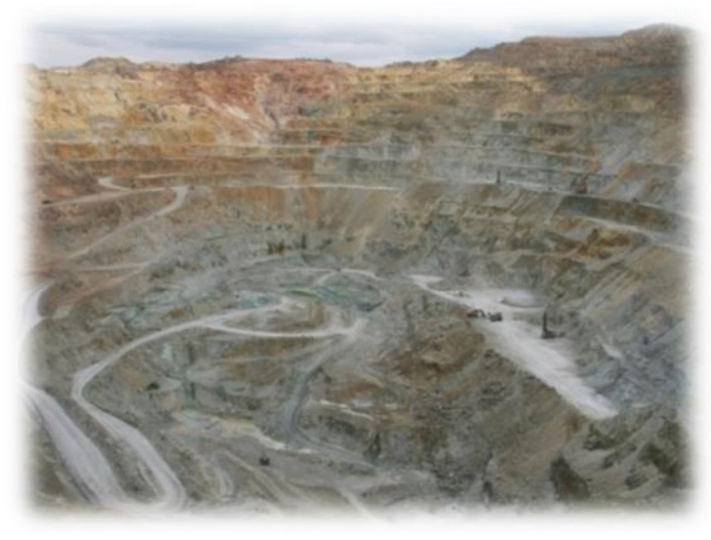

Fig.1. Ore quarry

\begin{tabular}{|r|r||r|r|}
\hline \begin{tabular}{r|r|r|}
$r i c$ \\
$x v i$
\end{tabular} & \multicolumn{1}{|r|}{ o qr o } & $\begin{array}{c}r c x \\
v i\end{array}$ & gad axsna \\
\hline 1 & - & 1 & 3736 \\
\hline 2 & - & 2 & 14216 \\
\hline 3 & - & 3 & 18357 \\
\hline 4 & - & 4 & 28807 \\
\hline 5 & - & 5 & 39422 \\
\hline 6 & - & 6 & 51907 \\
\hline 7 & 3034 & 7 & 62797 \\
\hline 8 & 8139 & 8 & 78941 \\
\hline 9 & 15840 & 9 & 78941 \\
\hline 10 & 15840 & 10 & 89391 \\
\hline 11 & 15840 & 11 & 89391 \\
\hline 12 & 15840 & 12 & 96211 \\
\hline
\end{tabular}

Fig.2. availability of gold in ore and without gold

As mentioned above, the proposed method was modeled in the LabView. fig.3-a shows the topology that is formed by an 800-point combination.

The coordinates of the points are formulated by random, evenly distributed numbers, and in image 3-b, corresponding to this topology, the distribution of the correlation dimension $\mathrm{D}(\varepsilon)$ depending on the distance $\varepsilon$. Abscissa axis is built on a logarithmic scale. Calculations are given at the initial value of $\varepsilon 0=0.1$, step spacing $\Delta \varepsilon=0.1$ the number of steps was 7 .

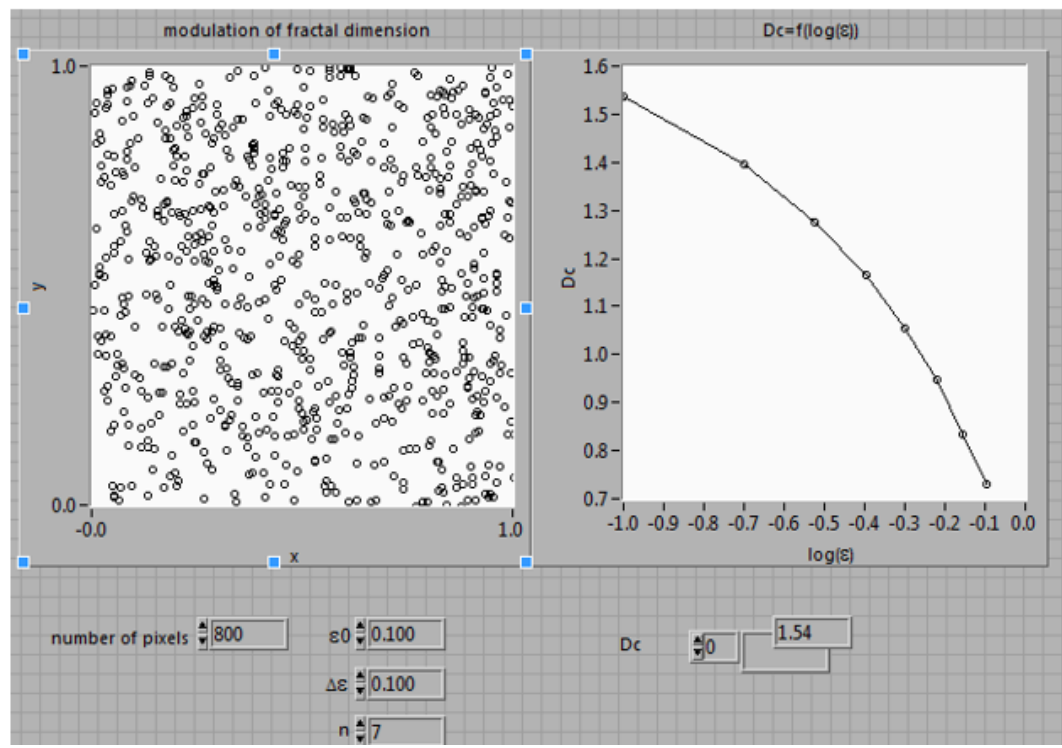

Fig.3. a topology formed by the coincidence of evenly distributed points and the distribution of the correlation dimension $D c(\varepsilon)$ depending on the distance $\varepsilon$ 
The experiment showed that:

1. Fractal dimension is less than Euclidean

2. As $\varepsilon(\varepsilon \rightarrow 1)$ increases, the fractal dimension of the collection of points decreases $D \varepsilon \rightarrow 0$. With increasing resolution, i.e. $\varepsilon \rightarrow 0$ the dimension tends to Euclidean $D_{F} \rightarrow \mathrm{D} \varepsilon \rightarrow D_{E}$

As a result of processing the actually presented data, the dimension turned out to be equal to $D_{F}=1,53$, therefore $\mathrm{H}=1 / 1,53 \approx 0,6$, which indicates the persistence of the measurement results, i.e., the possibility of predicting: in this case, an increased amount of quartzite (gold) in the ore, if much more ore is subjected to treatment over a longer observation time.

\subsection{Determination of fractal dimension using the Hurst normalized rane method}

Now let's turn to the definition of fractal dimension using the Hurst normalized method (the second method of determining Mandelbrot dimension).

Algorithm of $\mathrm{H}$ indicator estimation consists in the following:

Empty (t) describes a random process to be evaluated by Hurst's score.

1. We will designate through

$$
\langle\xi\rangle_{\tau}=\frac{1}{\tau} \sum_{t}^{\tau} \xi(\mathrm{t})
$$

$\langle\xi\rangle_{\tau}{ }_{-} \tau$ - average value.

2. We will designate through

$$
X(t, \tau)=\sum_{u=1}^{t}\left\{\xi(\mathrm{u})-\langle\xi\rangle_{\tau}\right\} X
$$

$\mathrm{X}(\mathrm{t}, \tau)$ - is the accumulated deviation from the average to the moment $\mathrm{t}$.

3. The difference between the maximum and minimum accumulated deviation is called the span - R.

$$
R(\tau)=\max _{1<t<\tau} X(t, \tau)-\min _{1<t<\tau} X(t, \tau)
$$

In this formula, $t$ is a discrete time taking an integer value, and $\tau$ is the duration of the time period under consideration.

4. The normalized span is defined as:

$$
\frac{R}{S}
$$

Where $\mathrm{S}$ is the standard deviation estimated by:

$$
S=\left(\frac{1}{\tau} \sum_{t=1}^{\tau}\left(\xi(t)-\langle\xi\rangle_{\tau}\right)^{2}\right)^{1 / 2}
$$

As Hurst discovered, the normalized span is very well described by the empirical ratio:

$$
\frac{R}{S}=\left(\frac{\tau}{2}\right)^{\mathrm{H}}
$$

from here:

$$
H=\frac{\log R / S}{\log \tau / 2}
$$

We conducted many experiments in this regard, which dealt with the change in the frequency of cardiorhythm and the part of seismography. Hurst score for cardiogram is 0.795 , for seismography 0.749 . Hurst algorithm representation graphs obtained for temperature change data are presented below.

The Hurts score makes it possible to associate fractals with the generalized Brownian movement [3] and thereby determine the presence in the process of persistence and anti-persistence, that is, predictability (deterministic) and its absence (anti-persistence), when it is impossible to predict the behavior of the process in the future (a completely random process).

So, when $\mathrm{H}<=1 / 2$, the correlation of past and future values is absent - the process is antipersistent when $\mathrm{H}>1 / 2$ maintains the current trend. The process becomes predictable - which is very important.

We have determined the $\mathrm{H}$ score for the processes showing the change in cardiorhythm frequency and seismogram segment. $\mathrm{H}=0.795$ for cardiorhythm frequency and for seismogram segment $\mathrm{H}=0.749$. There are presented graphs showing the implementation of the Hurst normalized span method for the temperature change process. 

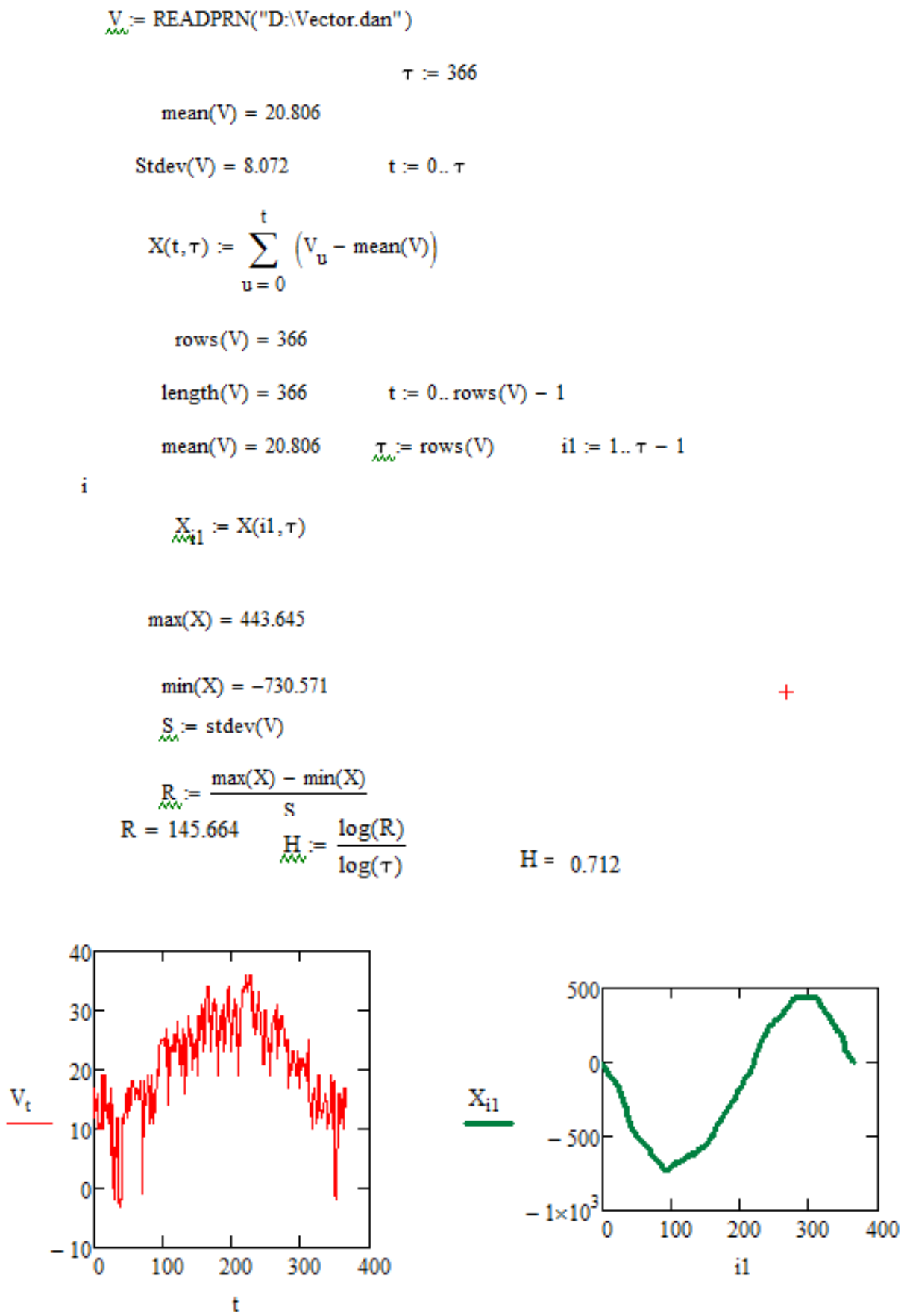

Fig.4. a) dependence of temperature on time; $b$ ) the accumulated deviation from the mean until the moment $t$

\section{Conclusions.}

As a result of the studies carried out, it can be concluded:

As a result of processing the actually presented ore data, the dimension was equal to $D_{F}=1,53$, therefore $\mathrm{H}=1 / 1,53 \approx 0,6$, which indicates the persistence of the measurement results, i.e., the possibility of predicting the behavior of the measurement object in the future (in this case, an increase in the amount of quartzite in the ore), if much more ore is subjected to treatment over a longer observation time.

The distribution of the correlation dimension Dc in relation to $\varepsilon$ showed that with the increase of $\varepsilon$, the fractal dimension $\left(D_{c} \rightarrow 0\right)$ of the collection of points decreases. In the process of decrease $(\varepsilon \rightarrow 0)$, the dimension of the set of points increases, tending to the value DE.

According to the second method, we have determined the $\mathrm{H}$ index for processes showing the change in cardiorhythm frequency and seismogram segment. $\mathrm{H}=0.795$ for cardiorhythm frequency and for seismogram segment $\mathrm{H}=0.712$. Graphs are presented showing the implementation of the Hurst 
normalized span method for the temperature change process. $\mathrm{H}=0.81$ The dimension is calculated by the expression: $\mathrm{D}_{\mathrm{F}}=2-\mathrm{H}$. This proves persistence in the measurement of dynamic processes.

A comparison of the above described methods has shown that the second method of determining fractal dimension is simpler, more obvious and relatively simple to implement. However, to do this, we must deal with data describing the dynamics of the process, that is, with a time series.

\section{REFERENCES}

1. J. Feder, "Fractals," Plenum Press, New York, 1988.

2. Chkheidze I., Otkhozoria N., Narchemashvili M. Evaluation of Measurement Quality Using the MonteCarlo Method // Universum: технические науки: электрон. научн. журн. 2021. 3(84).

3. Benoit B Mandelbrot. The fractal geometry of nature, San Francisco: W.H. Freeman, [1982], 460 p.

4. BROWN, Robert, Existence of active Molecules in Organic and Inorganic Bodies, The Philosophical Magazine, New Series, Vol. IV, No. 21, pp. 161-173.

5. Chkheidze I.M., Kharatishvili L.S., Karoyan I.B. "The modern concept of uncertainty of the result of measurement as a form of development and generalization of the concept of error." Works 11, Appendix to the Journal of the Academy of Educational Sciences of Georgia "Moambe," Tbilisi, 2008, 287-291 p.

6. J. Travis and J. Kring, "LabVIEW for Everyone Graphical Programming Made Easy and Fun," 3rd Edition, Prentice Hall, Berlin, 2006 